\title{
Probing of an Environmentally Friendly Regenerated Cellulose Material Having Bimorphic Behavior
}

\author{
Woo Sub Shim*, Jae Pil Kim ${ }^{1}$, Jung Jin Lee ${ }^{2}$, Joonseok Koh ${ }^{3}$, and Ik Soo Kim ${ }^{4}$ \\ Textile Engineering, Chemistry \& Science Department, North Carolina State University, Raleigh, NC 27695-8301, USA \\ ${ }^{1}$ Department of Materials Science and Engineering, Seoul National University, Seoul 151-742, Korea \\ ${ }^{2}$ Fiber System Engineering Program, Division of Polymer System Engineering, Dankook University, Yongin 448-701, Korea \\ ${ }^{3}$ Department of Textile Engineering, Konkuk University, Seoul 143-701, Korea \\ ${ }^{4}$ SK Chemicals, Gyeonggi-do 440-745, Korea
}

(Received December 18, 2007; Revised August 19, 2008; Accepted August 27, 2008)

\begin{abstract}
Novel regenerated cellulose material which was prepared from cellulose acetate fiber through the hydrolysis of acetyl groups have been developed by an environmentally friendly process without emitting toxic substances in addition to be at low production cost. They have composite crystalline structure constituted of cellulose II and cellulose IV. Also, they show a lamellar morphology with an increased amorphous region, as compared to conventional regenerated cellulose such as viscose rayon and cupra rayon. Our data obtained by several independent methods demonstrated that the adsorption properties of cellulose fibers depend predominantly on the amorphous region.
\end{abstract}

Keywords: Regenerated cellulose, Crystalline structure, Amorphous region, Viscose rayon, Cuprammonium rayon

\section{Introduction}

Cellulose is generally considered as being the most abundant and useful material, due to their excellent physical properties such as its gloss, specific gravity, and comfortable touch. Viscose rayon is a regenerated cellulosic fiber. The process involves dissolving wood pulp in an inorganic solvent containing sulfur and extruding the fiber. The Textile Fiber Products Identification Act (TFPIA) defines rayon as manufactured fibers composed of regenerated cellulose, as well as manufactured fibers composed of regenerated cellulose in which substituents have replaced the proton of the hydroxyl groups [1]. Rayon fibers are widely used in apparel, home furnishings, and industrial materials $[2,3]$.

Unfortunately, it was found that the regenerated cellulose fibers produced by conventional processes caused harmful environmental problem, posing their use in various applications to be diminished. In the typical manufacturing processes of these viscose rayon fibers, the raw material is alkali cellulose with a high $\alpha$-cellulose contents, which are prepared by treating wood pulp with an excess of sodium hydroxide solution. The alkali cellulose reacts with the $\mathrm{CS}_{2}$ to form xanthate ester groups. The carbon disulfide also reacts with the alkaline medium to form inorganic impurities which give the cellulose mixture a characteristic yellow color, and this material is referred to as yellow crumb. Because accessibility to the $\mathrm{CS}_{2}$ is greatly restricted in the crystalline regions of the soda cellulose, the yellow crumb is essentially a block copolymer of cellulose and cellulose xanthate. The raw materials such as sodium sulfate and zinc sulfide, which are used in the production of the rayon fibers, are subsequently recovered. But, carbon disulfide is recovered

*Corresponding author: jacob0720@gmail.com in varying degrees by larger plants; on the average, 30-35\% is recovered, the balance being lost through volatilization or decomposition during the spinning process. Although efforts by the major producers are expected to reduce carbon disulfide, increasing environmental concern has centered on the conventional preparation of conventionally regenerated cellulosic fibers since still some amount of the remaining carbon disulfide and heavy metal ions need to be recovered in waste treatment facilities at the plant to meet established water pollution regulations [4].

Because of the demand for pleasant environment materials without the release of pollutant, we developed a new cellulose material using environmentally friendly process. It was prepared from the untreated, cellulosic diacetate fiber with a degree of substituent of 2.0 or higher by saponifying (at $70-130{ }^{\circ} \mathrm{C}$ and preferably at $80{ }^{\circ} \mathrm{C}$ ) $75 \%$ or greater of the total acetyl groups of the cellulose acetate fiber into hydroxyl groups (Scheme 1) $[5,6]$. This new regenerated cellulose material is claimed to offer environmental advantages over other conventional regenerated cellulose fibers because it does not emit toxic materials such as carbon disulfide and heavy metal ions and enjoyed advantages of being very simple and low in production cost unlike conventional production methods of viscose rayon which use highly concentrated alkali solutions, carbon disulfide, and sulfuric acid. The structure of the new regenerated cellulose was investigated by FT-IR, X-ray diffraction, and birefringence measurement and its mechanical property and dyeing properties were also determined in this study.

\section{Experimental}

\section{Materials}

Viscose rayon fabric (plain weave, warp 82 filament/inch, 


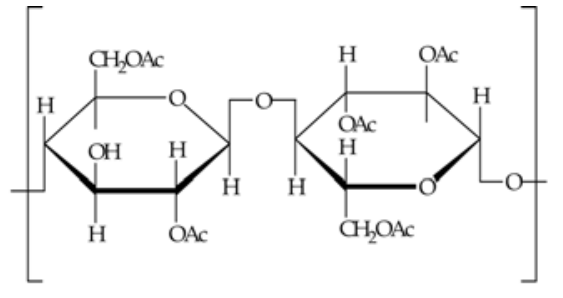

Amorphous acetate fiber

Scheme 1. Alkaline hydrolysis of cellulose acetate.

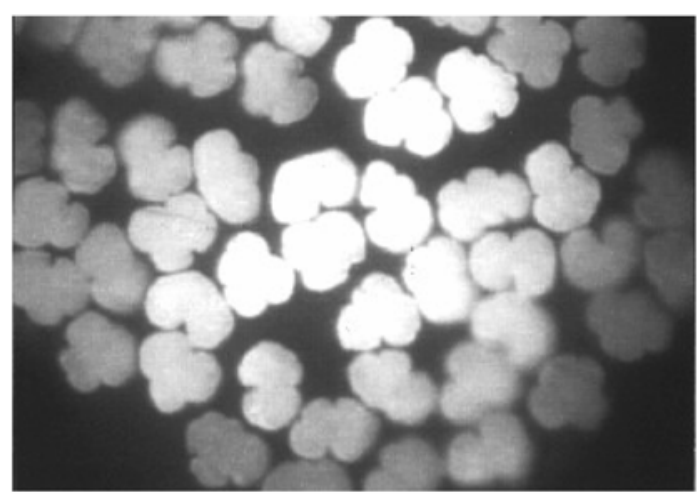

(a)

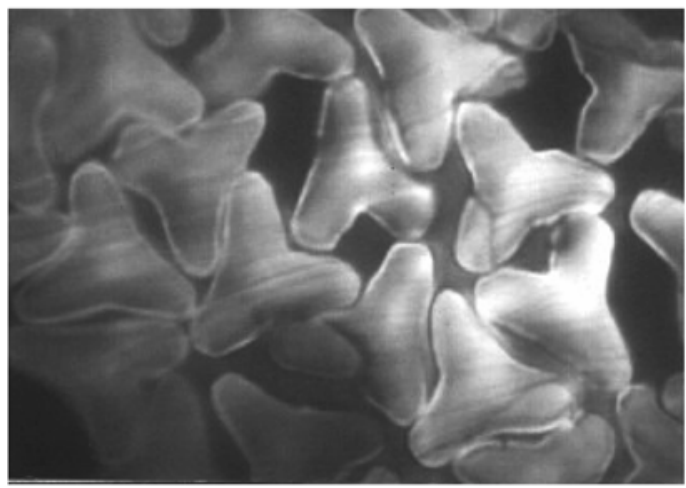

(b)

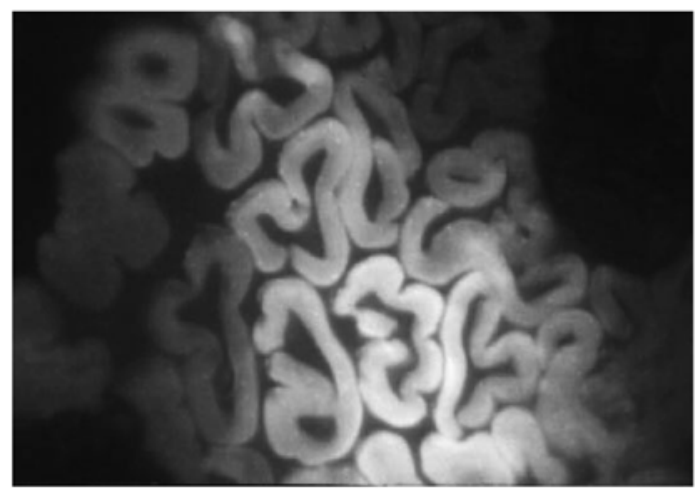

(c)

Figure 1. Various cross-sections of the new regenerated cellulose; (a) fine filament (75d/50f), (b) Y-shape filament (75d/33f), and (c) hollow filament (45d/33f).

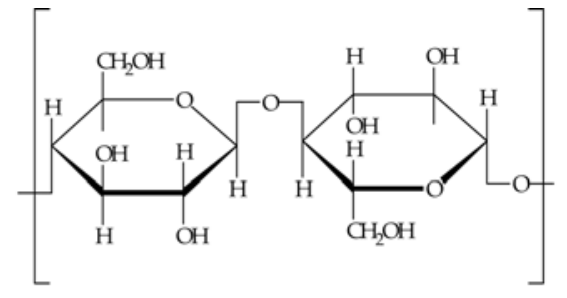

Crystalline new cellulose

weft 62 filament/inch), cupra rayon fabric (plain weave, Warp 86 filament/inch, weft 58 filament/inch), and a new regenerated cellulose fabric (plain weave, warp 96 filaments/ inch, weft 56 filaments/inch) obtained from cellulose acetate fibers by alkali-hydrolysis [5,6] were supplied courtesy of SK Chemicals (South Korea) (Figure 1). The three dyes used were commercial samples that were not purified prior to use; Everdirect Supra Red BWS (C.I. Direct Red 243), Procion H-E3B (C.I. Reactive Red 120), and Cibanone Red 2B (C.I. Vat Red 10). A commercial sample of a fixing agent (Neofix RP-70C, a polyethylene-based cationic resin) was supplied by Korea Fine Chemicals Co. (Daegu, Korea) for direct dyeing. A commercial sample of soaping agent (SNOGEN CS-940N, non-ionic) was supplied by Daeyoung Chemicals for reactive dyeing. Peregal $\mathrm{P}$, used as a leveling agent, was supplied by BASF and Setamol WS, a dispersing agent, and Dekol SN, a soaping agent, were supplied by Dae-Yang TexChem Co. Sodium hydrosulfite $\left(\mathrm{Na}_{2} \mathrm{~S}_{2} \mathrm{O}_{4}\right)$, used as a reducing agent and sodium hydroxide $(\mathrm{NaOH})$ were purchased from Sigma Aldrich Chemicals Co. for vat dyeing. All other reagents were of general purpose grade.

\section{Characterization}

FT-IR spectra were obtained with a Nicolet, Magna 7509 spectrometer using $\mathrm{KBr}$ pellet method to analyze new regenerated cellulose as well as viscose rayon and cupra rayon. Wide-angle X-ray diffraction (WAXD) was collected on a bundle of commercial viscose rayon, cupra rayon, and new regenerated cellulose at room temperature with a Bruker 2D-GADDS diffractometer using $\mathrm{Cu}-\mathrm{K}$ radiation with $\lambda=1.5418 \AA$ generated in a sealed $\mathrm{Cu}$ tube at $40 \mathrm{KV}$ and $40 \mathrm{~mA}$. Intensities were measured within the range of scattering angles $(2 \theta)$ of $3-33^{\circ}$ in the equatorial and meridional directions. The total scattering data was obtained by the summation of all the equatorial and meridional scattering data. The scattering data profiles were corrected for background and detector efficiency. The GADDS software integrated the intensity along each arc, making a correction for the effect of flat screen detection (unwarping), to create a one dimensional plot of intensity versus $2 \theta$, similar to a conventional powder-diffraction pattern. Birefringence values $(\Delta n)$ were obtained with a polarized microscope (Pol-12, Leica) having a 30 order Berek's 
compensator. The optical axis of a sample on the optical stage of the equipment is detected by rotating the sample in the plane. The detected axis gives the solution that satisfies $i_{2 w}=0$. In general, a positive birefringence $(\Delta n>0)$ indicates that the direction of the largest refractive index in the sample plane corresponds to the stretching direction. On the other hand, a negative birefringence $(\Delta n<0)$ means that the direction of the smallest refractive index in the plane corresponds to the stretching direction.

The tensile strength and elongation were determined with an extension rate of $10 \mathrm{~mm} / \mathrm{min}$ by the universal tensile testing instrument (RTM-1T, Yashima Works). Viscose rayon fiber (denier: 2.0 den, length: $51 \mathrm{~mm}$ ), cupra rayon fabric (denier: 2.0 den, length: $51 \mathrm{~mm}$ ), and a new regenerated cellulose fiber (denier: 2.0 den, length: $51 \mathrm{~mm}$ ) were tested either in dry state or in wet state at $25 \pm 2{ }^{\circ} \mathrm{C}$. Prior to testing in wet condition, all samples were neutralized in $1 \mathrm{~N} \mathrm{NaOH}$ for $15 \mathrm{~min}$ and rinsed thoroughly under tap.

The dyeings were carried out using a laboratory dyeing machine (Ahiba Spectradye Plus, Datacolor International) at liquor ratio $30: 1$, commencing at $30{ }^{\circ} \mathrm{C}$. The dyebath temperature was raised $1{ }^{\circ} \mathrm{C} / \mathrm{min}$. The dyeing methods used are shown in Figure 2, and their structures are shown in Table 1 . The fabrics dyed with direct, reactive, and vat dyes were rinsed and then treated with a fixing agent $(2.5 \%$ on the weight of fabric) for $15 \mathrm{~min}$ at $60{ }^{\circ} \mathrm{C}$ for direct dyeing and treated with a soaping agent $(0.5 \mathrm{~g} / l)$ for $30 \mathrm{~min}$ at $98{ }^{\circ} \mathrm{C}$ for reactive dyeing and then treated with hydrogen peroxide solution $(30 \% \mathrm{w} / \mathrm{v})$ for $15 \mathrm{~min}$ at $60{ }^{\circ} \mathrm{C}$ and next they were

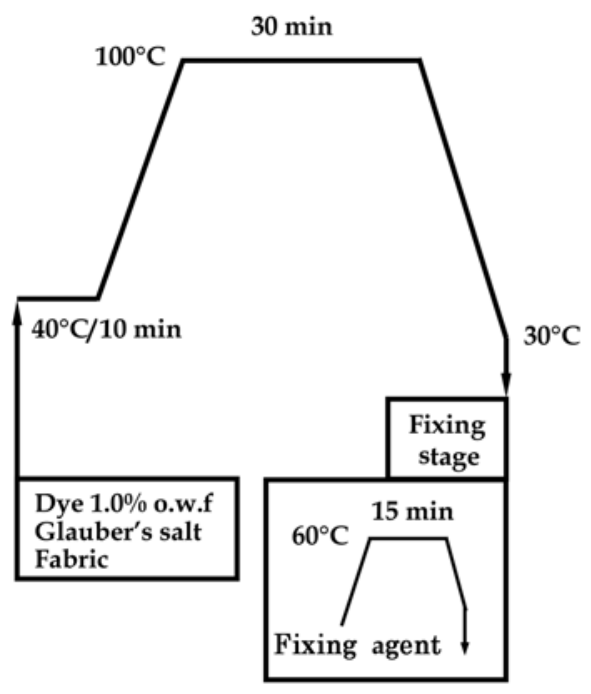

(a) Direct dyeing profile

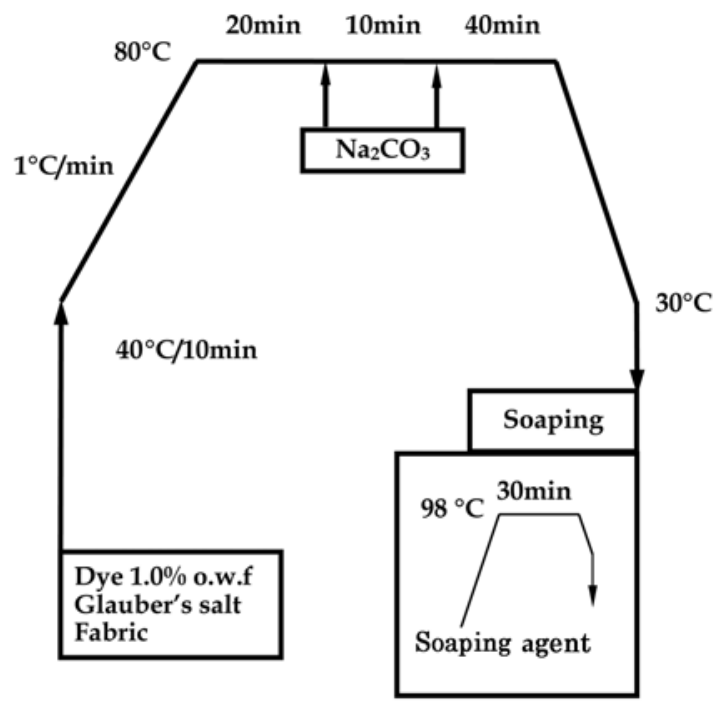

(b) Reactive dyeing profile

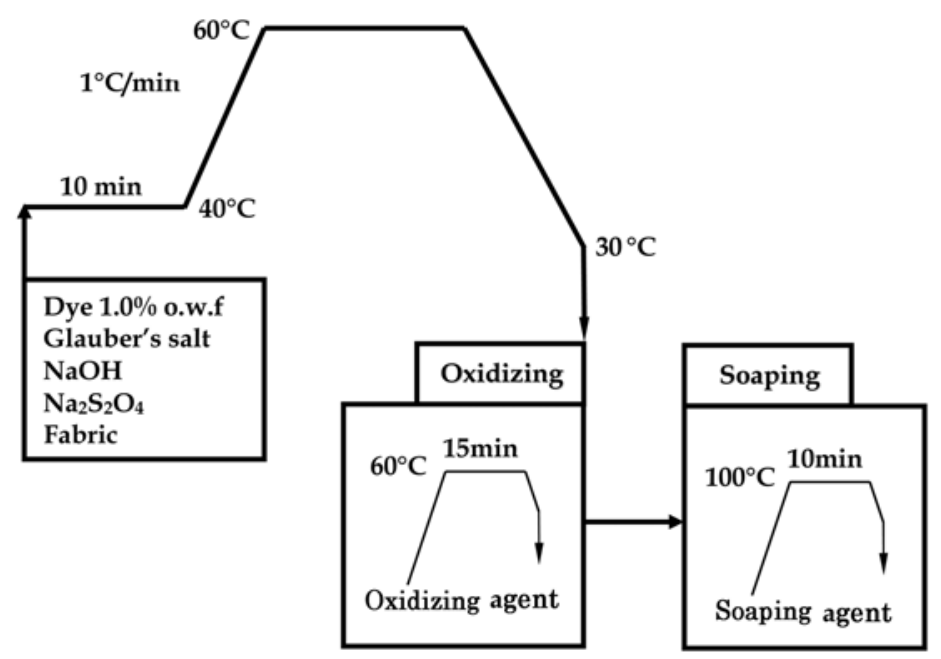

(c) Vat dyeing profile

Figure 2. Dyeing profile for three different dyes. 
Table 1. Structures of the dyes used in this study

Dyes
C.I. Direct
Red 243
Red 120
Red 10

soaped in the aqueous solution of $2.5 \mathrm{~g} / l$ Dekol $\mathrm{SN}$ for 15 min at the boil for vat dyeing.

Color strength $(K / S)$ of the dyed samples was measured using a Datacolor Spectroflash SF 600 Plus-CT spectrophotometer with following: illuminant D65, large area view, specular component included, and CIE 1964 Supplemental Standard Observer (10 degree observer) by using equation (1). With each sample folded twice, reflectance was measured four times at different surfaces and averaged.

$$
\frac{K}{S}=\frac{(1-R)^{2}}{2 R}
$$

where $R$ is the reflectance of an infinitely thick layer of the material illuminated with light of a known wavelength, $K$ is the absorption coefficient, and $S$ is the scattering coefficient. The function of $K / S$ is directly proportional to the concentration of the colorant in the substrate. The fabrics were dyed with these dyes with $0.5,1.0,2.0,4.0$, and $6.0 \%$ o.w.f. dye concentration for evaluating the build-up property.

\section{Results and Discussion}

WAXD curves are shown in Figure 3 for the regular viscose rayon fibers (a), cupra rayon (b), and new regenerated cellulose (c). All the data contained almost the same features: an amorphous halo with several crystalline Bragg peaks. Crystallinity can be determined from a wideangle X-ray diffraction (WAXD) scan by comparing the area under the crystalline peaks to the total scattered intensity. The intense amorphous halo in all the data indicates the existence of a significant amount of amorphous, disordered phase. The numbers and positions of the Bragg peaks in all the patterns are almost similar, indicating that the crystalline structures of all the fibers are similar. All reflection peaks can be indexed based on the known cellulose II crystal structure [7]. The higher amorphous content and poor crystalline orientation of new regenerated cellulose fiber as compared with viscose and cupra rayons may arise from the saponification process of cellulose acetate which is achieved by immersing it in the aqueous alkaline solution at $80^{\circ} \mathrm{C}$ $[5,6]$. This may be due to the amorphous regions of the cellulose diacetate fibers used as the starting material.

As seen in Figure 3, the non-crystalline part was taken as contributing to the intensity of the diffuse background and the crystalline peaks as contributing to the intensity of the selectively diffracted radiation (the peaks occurring on top of the background). All the amorphous backgrounds and the Bragg peaks were resolved by the curve fitting method. In all the data, we found three Bragg peaks at $2 \theta \approx 12^{\circ}, 19^{\circ}$, and $22^{\circ}$ which are indexed as $(110),(110)$, and (020) peaks of cellulose II [7]. It has been known that conventional rayon fibers, such as viscose rayon, cupra rayon, Bemberg rayon, high tenacity rayon, and Fortisan have a crystalline structure of cellulose II [8]. In Figure 3 (c), we found another peak $2 \theta$ $\approx 15.4^{\circ}$ which is (110) peak of cellulose IV phase [9]. The peak at $2 \theta \approx 22^{\circ}$ was attributed to $(020) /(200)$ peak of cellulose IV which overlapped with (020) peaks of cellulose II $[7,9]$. The coexistence of the Bragg peaks from cellulose II and IV for new regenerated cellulose indicates bimorphic behavior of the crystalline structure. It was also suggested that the tensile properties of cellulose were affected by the type of crystal structure.

Since most crystalline Bragg peaks of viscose rayon, cupra rayon, and new regenerated cellulose fibers were located in the region of $2 \theta \leq 50^{\circ}$, their approximate apparent crystallinities were estimated based on the curve fitted data 


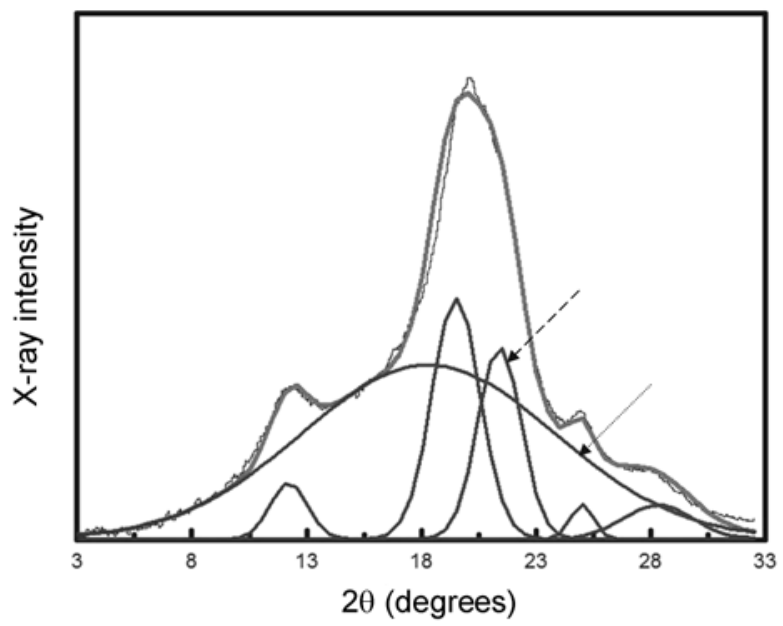

(a) Viscose rayon

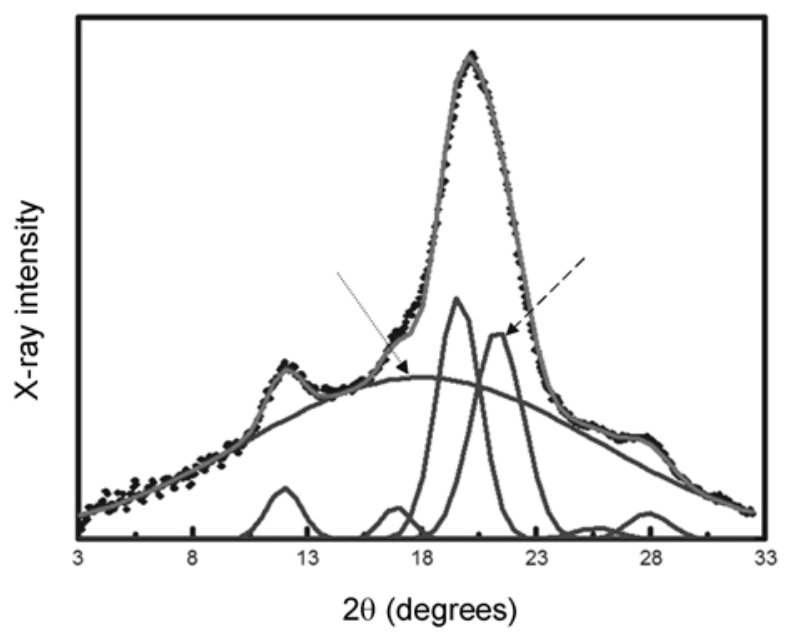

(b) Cupra rayon

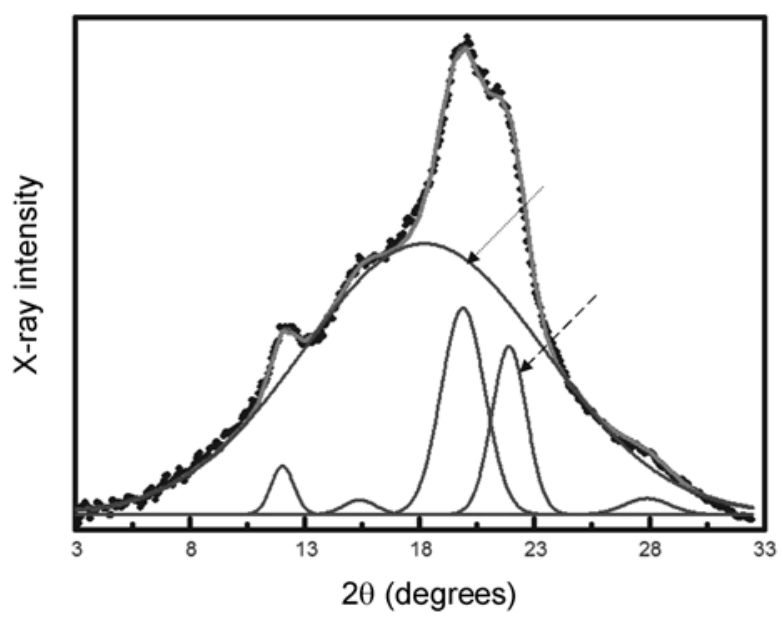

(c) New regenerated cellulose

Figure 3. Wide angle X-ray scattering pattern resolved into individual integral intensities of the corresponding crystal planes and the amorphous phase. ( \........ ) amorphous component, ( 4 ------) crystalline component.

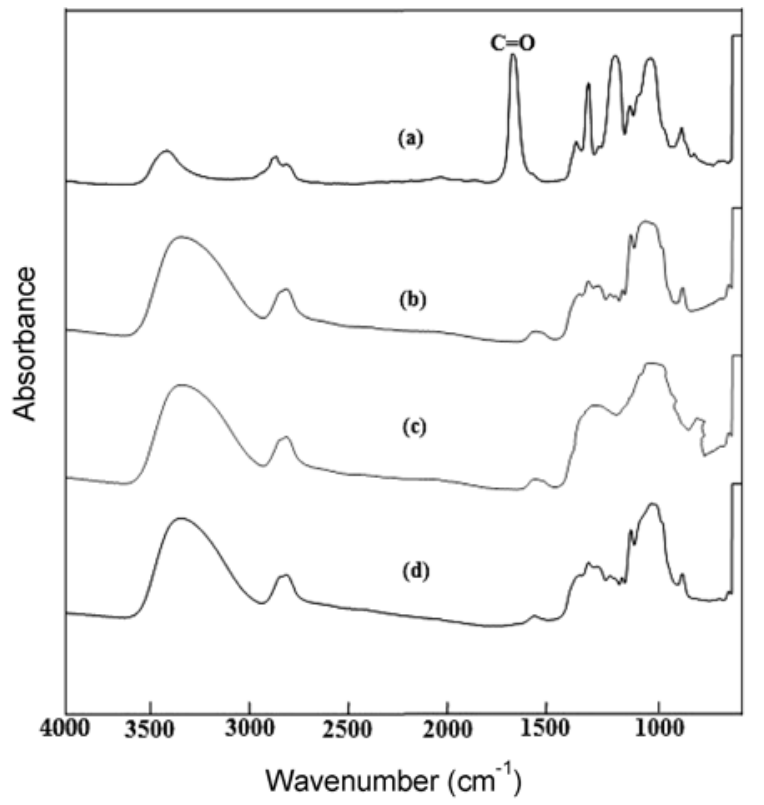

Figure 4. FT-IR spectra of (a) cellulose diacetate, (b) viscose rayon, (c) cupra rayon, and (d) new regenerated cellulose.

shown in Figure 3. The estimated values of crystallinity were $36 \pm 2 \%, 51 \pm 2 \%$, and $28 \pm 2 \%$ for viscose rayon, cupra rayon, and new regenerated cellulose, respectively. The lower value of crystallinity of new regenerated cellulose can exhibit better solvent processing characteristics and dyeability.

Figure 4 shows FT-IR spectra of cellulose acetate, viscose rayon, cupra rayon, and new regenerated cellulose. The deacetylation of the cellulose diacetate fiber was indentified through FT-IR spectra. In these data, the C-O strectching peak of the $\beta$-1,4-linked D-glucopyranose was read at 1160 $\mathrm{cm}^{-1}$ and the carbonyl band of the acetyl groups were read at $1760 \mathrm{~cm}^{-1}$ [10]. These FT-IR peaks were calculated by integration and the ratio between them was obtained. The large quantity of carbonyl groups in the acetate moieties produced the large peak at $1760 \mathrm{~cm}^{-1}$ in curve (a) corresponds to the untreated cellulose diacetate, whereas the carbonyl peak at $1760 \mathrm{~cm}^{-1}$ was greatly reduced and almost disappeared in curve (d). On the basis of the FT-IR data, it was confirmed that almost the entire acetyl group of the cellulose diacetate was substituted with a hydroxyl group and that the cellulose was completely regenerated by ecofriendly process.

The measured tensile strength and elongation at the break of samples is shown in Figures 5 and 6 . The samples were first tested in the dry state. For the new regenerated cellulose, the tensile strength was 1.27 (gf/den) and the elongation was $35 \%$. They have lower tensile strength and higher elongation than viscose rayon ( $1.55 \mathrm{gf} / \mathrm{den}, 15.3 \%)$ and cupra rayon (2.63 gf/den, $13.2 \%)$. Next, samples were tested in the wet state. The results showed a significant 


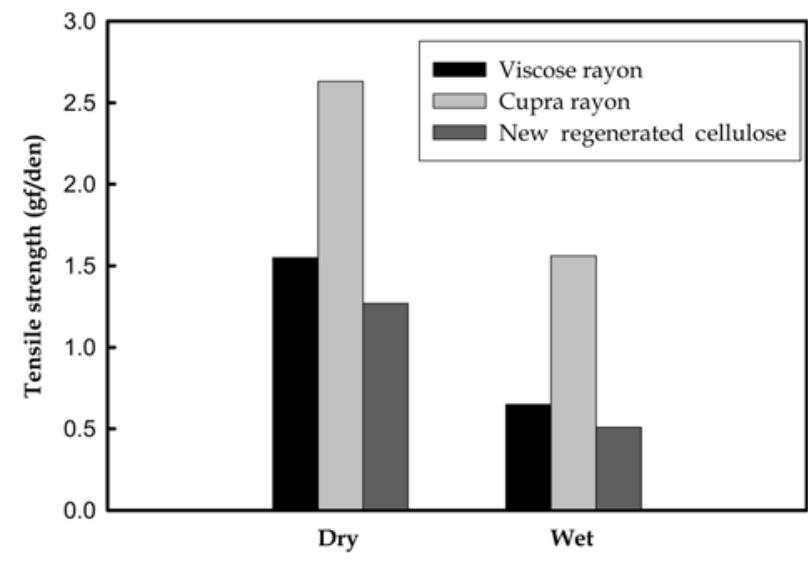

Figure 5. The tensile strength of viscose rayon, cupra rayon, and new regenerated cellulose.

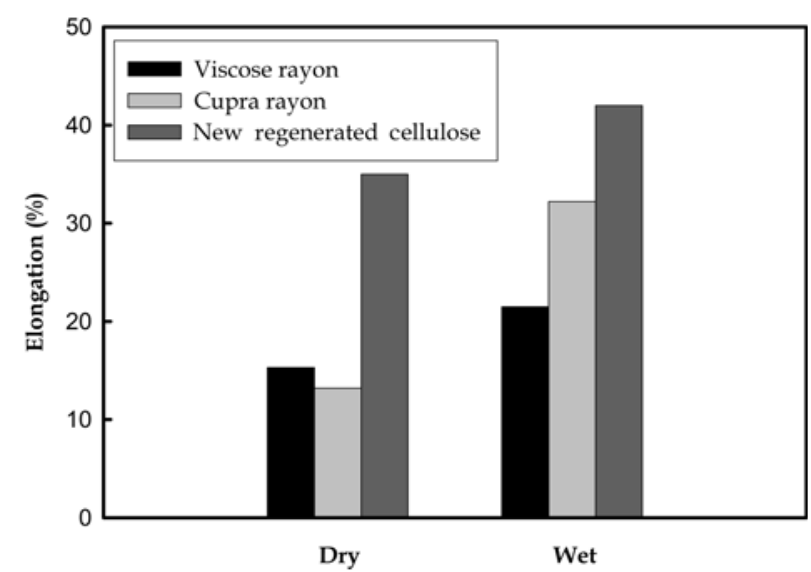

Figure 6. The elongation of viscose rayon, cupra rayon, and new regenerated cellulose.

difference in the mechanical properties relative to the dry state. For the new regenerated cellulose, the tensile strength was 0.51 (gf/den) and the elongation was $42 \%$. They have a lower tensile strength and higher elongation than viscose rayon $(0.65 \mathrm{gf} / \mathrm{den}, 21.5 \%)$ and cupra rayon $(1.56 \mathrm{gf} / \mathrm{den}$, $32.2 \%)$.

Figure 7 shows build-up properties of three different dyes on viscose rayon, cupra rayon, and new regenerated cellulose. The build-up properties of the new regenerated cellulose are better than those of viscose rayon and cupra rayon for the three different dyes. The results show that new regenerated cellulose dyes to a much deeper shade than the other rayons. The dyeing properties of fibrous polymeric materials depend on the relative amount of amorphous phase and the chain packing, especially in the intermediate phase between crystalline and amorphous phases. All the structural parameters of solvent and dye molecules strongly affect the sorption characteristics of fibrous polymers. Since the molecular orientation provides information on the degree of chain packing in all the crystalline and intermediate phases,

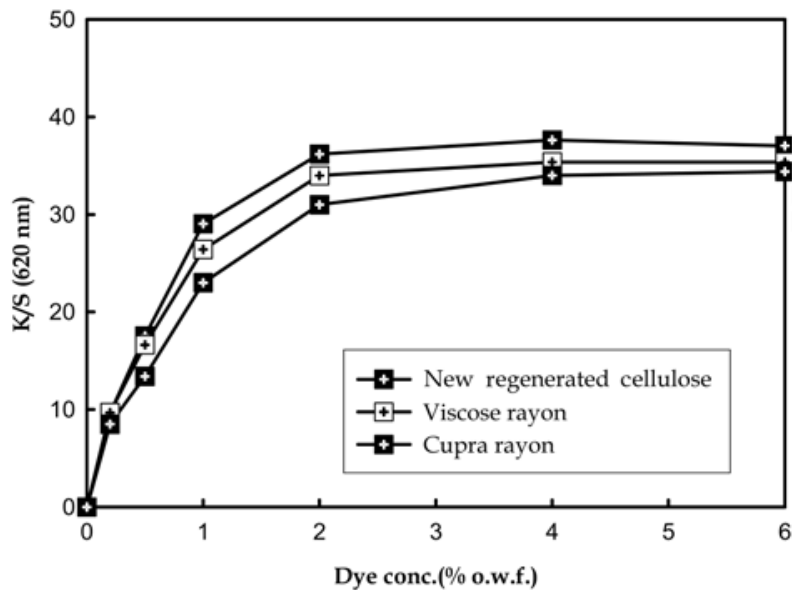

(a)

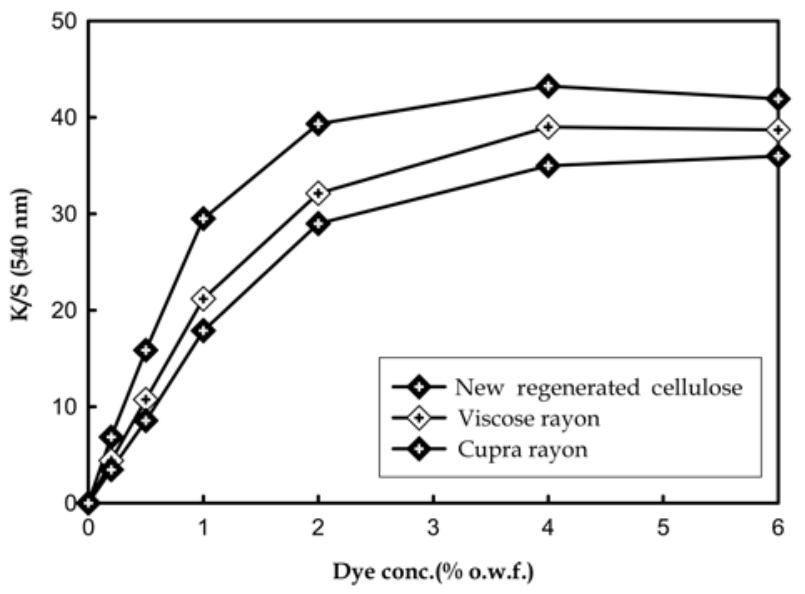

(b)

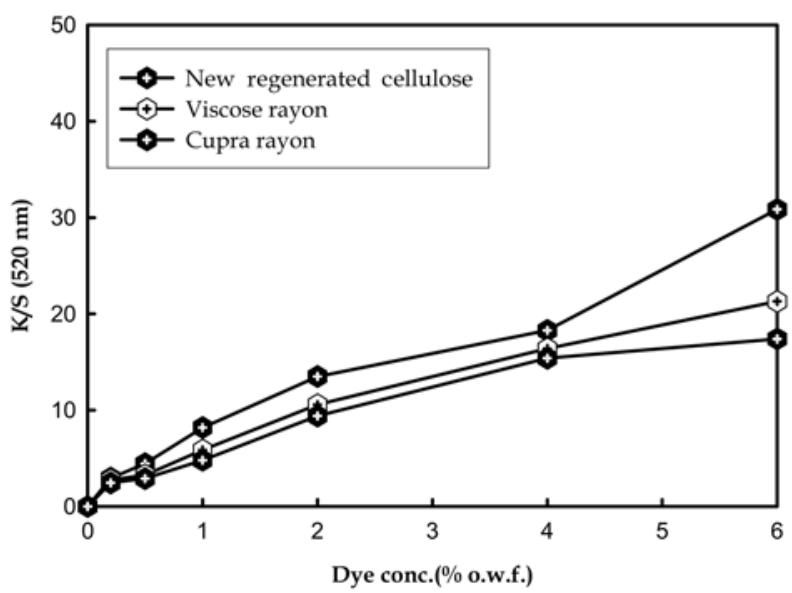

(c)

Figure 7. Build-up properties of three different dyes on viscose, cupra, and new regenerated cellulose; (a) C.I. Direct Red 243, (b) C.I. Reactive Red 120, and (c) C.I. Vat Red 10.

the birefringence measurement was conducted to measure the degree of orientation of viscose, cupra, and new 
regenerated cellulose materials. This resulted in a phase difference or retardation $(\tau)$ as the light traverses the sample material. If the material is not dichroic, then the retardation is related directly to its birefringence [11]. Birefringence data, determined by a polarized microscope, were found to support the WAXD results; birefringence of viscose rayon (0.036) and cupra rayon (0.038) was higher than that of new regenerated cellulose material $(0.019)$. These results mean the chain packing of viscose rayon and cupra rayon is better than that of new regenerated cellulose fiber, which points out poor dyeability [12].

\section{Conclusion}

We investigated new environmentally friendly regenerated cellulose which has coexisting cellulose II and IV prepared by saponifying at least $75 \%$ of the total acetyl groups of a cellulose acetate fiber with a degree of substitution of 2.0 or higher into hydroxyl groups. The mechanical property is found to be similar to those of already commercialized viscose and cupra rayon and dyeing property is slightly better than those of other regenerated cellulose.

\section{Acknowledgements}

The authors appreciate the financial support from the Graduate Student Support Plan (GSSP) in the college of Textiles, NC State University, USA and the Ministry of Commerce, Industry and Energy, South Korea (Support for Industrial Technology Development Program, No. D11-08007).

\section{References}

1. K. Kong, R. J. Davies, M. A. McDonald, R. J. Young, M. A. Wilding, R. N. Ibbett, and S. J. Eichhorn, Biomacromolecules, 8, 624 (2007).

2. S. Klahorst, A. Kumar, and M. M. Mullins, Text. Chem. Color., 26, 13 (1994).

3. F. Jones in "The Theory Coloration of Textiles" (A. Johnson Ed.), pp.405-409, Society of Dyers and Colourists, Bradford, 1989.

4. K. Bredereck and F. Hermanutz, Rev. Prog. Coloration, 35, 59 (2005).

5. I. S. Kim, J. S. Ahn, and B. H. Kim, Kor. Patent, 0015443 (2000).

6. I. S. Kim, J. S. Ahn, and B. H. Kim, U. S. Patent, 6361862 (2000).

7. F. Kolpak and J. Blackwell, Macromolecules, 9, 273 (1976).

8. W. E. Moriton and J. W. S. Hearle, "Physical Properties of Textile Fibers", pp.84-94, Butterworth, London, 1962.

9. E. S. Gardiner and A. Sarko, Can. J. Chem., 63, 173 (1985).

10. A. Carrillo, X. Colom, J. J. Sunol, and J. Saurina, Eur. Polym. J., 40, 2229 (2004).

11. R. M. A. Azzam and N. M. Bashara, "Ellipsometry and Polarized Light", pp.52-67, North Holland, Amsterdam, 1979.

12. J. Koh, I. S. Kim, S. S. Kim, W. S. Shim, and J. P. Kim, Fiber. Polym., 5, 44 (2004). 